\title{
Almost classical solutions of Hamilton-Jacobi equations
}

\section{Robert Deville and Jesús A. Jaramillo}

\begin{abstract}
We study the existence of everywhere differentiable functions which are almost everywhere solutions of quite general HamiltonJacobi equations on open subsets of $\mathbb{R}^{d}$ or on $d$-dimensional manifolds whenever $d \geq 2$. In particular, when $M$ is a Riemannian manifold, we prove the existence of a differentiable function $u$ on $M$ which satisfies the Eikonal equation $\|\nabla u(x)\|_{x}=1$ almost everywhere on $M$.
\end{abstract}

\section{Introduction}

It has been proved by Z. Buczolich [4] that if $d \geq 2$, there exists $u: \mathbb{R}^{d} \rightarrow \mathbb{R}$, differentiable at every point, such that $\nabla u(0)=0$ and $\|\nabla u(x)\| \geq 1$ almost everywhere, thus giving a negative answer to the gradient problem of C. E. Weil [10]. Malý and Zelený [8] gave an elegant proof of this result using a new mathematical game. Then Deville and Matheron [6], refining the methods introduced by the above authors, proved that if $\Omega$ is a bounded open subset of $\mathbb{R}^{d}$ with $d \geq 2$, there exists a function $u: \bar{\Omega} \rightarrow \mathbb{R}$, continuous on $\bar{\Omega}$, differentiable at every point of $\Omega$, such that $u(x)=0$ for all $x \in \partial \Omega$, and such that $\|\nabla u(x)\|=1$ almost everywhere on $\Omega$. Notice that because of Rolle's theorem, there exists $x_{0} \in \Omega$ such that $\nabla u\left(x_{0}\right)=0$, so the function $u$ cannot be $\mathcal{C}^{1}$-smooth. We shall call $u$ an almost-classical solution of the Eikonal equation $\|\nabla u\|=1$. This equation has also a unique viscosity solution, which is the function $x \mapsto \operatorname{dist}(x, \partial \Omega)$, where $\operatorname{dist}(x, \partial \Omega)=\inf \{\|x-y\| ; y \in \partial \Omega\}$. The viscosity solution is not everywhere differentiable on $\Omega$. Therefore, an almost classical solution of the Eikonal equation is not equal to the viscosity solution of the Eikonal equation. Nevertheless in optimal control, where

2000 Mathematics Subject Classification: 26B05, 35B65, 58J32.

Keywords: Hamilton-Jacobi equations; eikonal equation on manifolds; almost everywhere solutions. 
this equation arises naturally, the viscosity solution is the "right" solution of the Eikonal equation. We refer to [2] and [5] for an account on viscosity solutions of Hamilton-Jacobi equations.

The contents of the paper are as follows. In Section 2, we recall some technical results from [6] which will be needed in this paper. In Sections 3 and 4 , we study the existence of almost-classical solutions for more general Hamilton-Jacobi equations on open subsets of $\mathbb{R}^{d}$. Finally, Section 5 is devoted to Hamilton-Jacobi equations on manifolds, and in particular we will consider the Eikonal equation $\|\nabla u(x)\|_{x}=1$ on a Riemannian manifold. See e.g. [1], [7] and [9] for further information about Hamilton-Jacobi equations on Riemannian manifolds.

Now we introduce some terminology. Let $\Omega$ be an open subset of $\mathbb{R}^{d}$, and let $F: \mathbb{R} \times \Omega \times \mathbb{R}^{d} \rightarrow \mathbb{R}$ and $u_{0}: \partial \Omega \rightarrow \mathbb{R}$ be continuous. As usual, we say that a continuous function $u: \bar{\Omega} \rightarrow \mathbb{R}$ is a classical solution of $F(u(x), x, \nabla u(x))$ $=0$ with Dirichlet condition $\left.u\right|_{\partial \Omega}=u_{0}$ if for all $x \in \partial \Omega, u(x)=u_{0}(x)$, and for all $x \in \Omega, u$ is differentiable at $x$ and $F(u(x), x, \nabla u(x))=0$.

We say that $u$ is a classical subsolution of $F(u(x), x, \nabla u(x))=0$ if for all $x \in \Omega, u$ is differentiable at $x$ and $F(u(x), x, \nabla u(x)) \leq 0$.

Definition 1.1. We say that a continuous function $u: \bar{\Omega} \rightarrow \mathbb{R}$ is an almost classical solution of $F(u(x), x, \nabla u(x))=0$ with Dirichlet condition $\left.u\right|_{\partial \Omega}=u_{0}$ if:

- $u(x)=u_{0}(x)$ for all $x \in \partial \Omega$,

- $u$ is a classical subsolution of $F(u(x), x, \nabla u(x))=0$,

- and $u$ satisfies $F(u(x), x, \nabla u(x))=0$ for almost every $x \in \Omega$ (in the sense of Lebesgue measure on $\mathbb{R}^{d}$ ).

Notice that a classical solution is an almost classical solution, and that if $u$ is an almost classical solution, then $u$ is continuous on $\bar{\Omega}$ and differentiable at every point of $\Omega$. In many natural examples, classical solutions of the Hamilton-Jacobi equation $F(u(x), x, \nabla u(x))=0$ exist only under very restrictive conditions on $F$. We prove the existence of almost classical solutions under quite general hypotheses on $F$. Observe that our results imply the existence of an almost classical solution $u$ of the Eikonal equation satisfying the boundary condition $\left.u\right|_{\partial \Omega}=0$. In particular, we have:

Theorem 1.2. Let $\Omega$ be an open subset of $\mathbb{R}^{d}$ with $d \geq 2$, and let $F$ : $\Omega \times \mathbb{R}^{d} \rightarrow \mathbb{R}$ be a continuous function. Suppose that the following conditions hold:

(A) There exists a continuous function $u_{0}: \bar{\Omega} \rightarrow \mathbb{R}$, which is $\mathcal{C}^{1}$-smooth on $\Omega$ and such that $F\left(x, \nabla u_{0}(x)\right) \leq 0$, for every $x \in \Omega$. 
(B) For each compact subset $K \subset \Omega$, there exists $M_{K}>0$ such that

$$
\inf \left\{F(x, p): x \in K, p \in \mathbb{R}^{d},\|p\| \geq M_{K}\right\}>0 .
$$

Then there exists an almost classical solution of $F(x, \nabla u(x))=0$, with Dirichlet condition $\left.u\right|_{\partial \Omega}=u_{0}$.

The above result will actually follow from the more general Theorem 3.1 that will also provide other existence results of almost classical solutions of Hamilton-Jacobi equations. The proof of Theorem 3.1 will be given in Section 4.

In the last section, we consider Hamilton-Jacobi equations defined on a smooth manifold $M$ of dimension $d \geq 2$, which always will be assumed to be Hausdorff and second countable. As usual, $T M$ denotes the tangent bundle of $M$. A point in $T M$ will be $(x, v)$, where $x \in M$ and $v$ belongs to the tangent space $T_{x} M$. In the same way, $T^{*} M$ denotes the cotangent bundle of $M$. A point in $T^{*} M$ will be $(x, \xi)$, where $x \in M$ and $\xi \in T_{x}^{*} M$ is a linear form on the tangent space $T_{x} M$. If $u: M \rightarrow \mathbb{R}$ is differentiable at $x \in M$ we denote its differential at $x$ by $d u(x)$. Under suitable hypotheses on $F: T^{*} M \rightarrow \mathbb{R}$, we obtain the existence of almost classical solutions of an equation of the form $F(x, d u(x))=0$. In particular, we obtain:

Theorem 1.3. Let $M$ be a smooth manifold of dimension $d \geq 2$, and let $F: T^{*} M \rightarrow \mathbb{R}$ be a $\mathcal{C}^{1}$-smooth function. Suppose that the following conditions hold:

(A) There exists a $\mathcal{C}^{1}$ function $u_{0}: M \rightarrow \mathbb{R}$ such that $F\left(x, d u_{0}(x)\right) \leq 0$, for every $x \in M$.

(B) For each $x \in M$, the set $B(x)=\left\{\xi \in T_{x}^{*} M: F(x, \xi) \leq 0\right\}$ is compact, the set $S(x)=\left\{\xi \in T_{x}^{*} M: F(x, \xi)=0\right\}$ is connected, and the function $F(x, \cdot)$ has maximal rank on the set $S(x)$.

Then there exists a differentiable function $u: M \rightarrow \mathbb{R}$ such that $F(x, d u(x))=0$ for almost every $x \in M$.

If now we have a Riemannian manifold $(M, g)$ and $u: M \rightarrow \mathbb{R}$ is differentiable, for every $x \in M$ we identify in the usual way the differential $d u(x)$ with the gradient $\nabla u(x)$ by means of the scalar product $g_{x}(\cdot, \cdot)$ on the tangent space $T_{x} M$. In this case we obtain the following analogue of Theorem 1.2:

Theorem 1.4. Let $(M, g)$ be a Riemannian manifold of dimension $d \geq 2$, and let $F: T M \rightarrow \mathbb{R}$ be a continuous function. Suppose that the following conditions hold: 
(A) There exists a $\mathcal{C}^{1}$ function $u_{0}: M \rightarrow \mathbb{R}$, such that $F\left(x, \nabla u_{0}(x)\right) \leq 0$, for every $x \in M$.

$(B)$ There exists a locally bounded function $\rho: M \rightarrow(0, \infty)$ such that, for every $x \in M$, the set $B(x)=\left\{v \in T_{x} M: F(x, v) \leq 0\right\}$ is contained in the ball of center 0 and radius $\rho(x)$ in $T_{x} M$.

Then there exists a differentiable function $u: M \rightarrow \mathbb{R}$ such that $F(x, \nabla u(x))=0$ for almost every $x \in M$.

Thus if for a Riemannian manifold $(M, g)$ we consider the function $F$ : $T M \rightarrow \mathbb{R}$ given by

$$
F(x, v)=\|v\|_{x}-1=\left(g_{x}(v, v)\right)^{1 / 2}-1,
$$

it is clear that the constant functions $u_{0} \equiv 0$ and $\rho \equiv 1$ satisfy the above requirements. Therefore we obtain that there exists a differentiable function $u$ on $M$ which satisfies the Eikonal equation $\|\nabla u(x)\|_{x}=1$ almost everywhere on $M$. Whenever the manifold $M$ is compact, there exists a point $x_{0} \in M$ such that $\nabla u\left(x_{0}\right)=0$. Therefore, there is no classical solution of this equation, and an almost classical solution $u$ of this equation cannot be $\mathcal{C}^{1}$-smooth. So almost classical solutions of Hamilton-Jacobi equations are often exotic.

\section{Preliminary results}

We recall three lemmas from [6] that we shall use here. The first lemma is a criterium of differentiability for the sum of a series of $\mathcal{C}^{1}$-smooth functions. We shall use the following notation: if $X$ and $Z$ are Banach spaces and $f: X \rightarrow Z$, then the oscillation of $f$ with respect to $\delta>0$ is defined by

$$
o s c(f, \delta)=\sup \left\{\left\|f\left(x_{1}\right)-f\left(x_{2}\right)\right\|: x_{1}, x_{2} \in X,\left\|x_{1}-x_{2}\right\| \leq \delta\right\}
$$

Lemma 2.1. Let $\left(u_{n}\right)_{n \geq 1}$ be a sequence of $\mathcal{C}^{1}$ functions between two Banach spaces $X$ and $Y$. Assume that:

(a) the series $\left(\sum \nabla u_{n}(x)\right)$ is pointwise convergent;

(b) the sequence $\left(\nabla u_{n}\right)$ converges uniformly to 0 ;

(c) $\left\|u_{n+1}\right\|_{\infty}=o\left(\left\|u_{n}\right\|_{\infty}\right)$;

(d) $\lim _{n \rightarrow \infty} \operatorname{osc}\left(\sum_{k=1}^{n} \nabla u_{k},\left\|u_{n+1}\right\|_{\infty}\right)=0$.

Then the series $\left(\sum u_{n}\right)$ is uniformly convergent, the function $u:=\sum_{n=1}^{\infty} u_{n}$ is everywhere differentiable, and $\nabla u(x)=\sum_{n=1}^{\infty} \nabla u_{n}(x)$ for all $x \in X$. 
We say that a subset $Q$ of $\mathbb{R}^{d}$ is a cube if $Q=\prod_{i=1}^{d}\left[a_{i}, b_{i}[\right.$, where each $\left[a_{i}, b_{i}\right]$ is a closed and bounded interval of $\mathbb{R}$. And we say that $Q$ is a closed cube if $Q=\prod_{i=1}^{d}\left[a_{i}, b_{i}\right]$. A function $v$ defined on a cube $Q$ is said to be piecewise constant if there is a finite partition $\mathcal{Q}$ of $Q$ into cubes such that $v$ is constant on every cube of the partition $\mathcal{Q}$. The following result gives the existence of a $\mathcal{C}^{\infty}$-smooth function $u: \mathbb{R}^{d} \rightarrow \mathbb{R}$, which vanishes in a neighbourhood of the exterior of a cube $Q$ and such that its derivative is equal to $a$ or $-a$ (where $a$ is a given non zero vector in $\mathbb{R}^{d}$ ) on a subset of $Q$ of measure almost equal to the measure of $Q$. The Lebesgue measure on $\mathbb{R}^{d}$ will be denoted $\lambda_{d}$.

Lemma 2.2. Let $a \in \mathbb{R}^{d}$ be a non zero vector, let $Q$ be a cube in $\mathbb{R}^{d}$, and let $\varepsilon>0$. Then, there exists a bounded, $\mathcal{C}^{\infty}$-smooth function $u: \mathbb{R}^{d} \rightarrow \mathbb{R}$ satisfying the following properties:

(a) $u$ vanishes in a neighbourhood of $\partial Q$ and $\|u\|_{\infty} \leq \varepsilon$;

(b) $\lambda_{d}(\{x \in Q: \nabla u(x)=-a$ or $\nabla u(x)=a\}) \geq(1-\varepsilon) \lambda_{d}(Q)$;

(c) one can write $\nabla u=v+w$ with $\|w\|_{\infty}<\varepsilon$; the set $\{v(x): x \in Q\}$ is included in the segment $[-a, a]$, and the function $v$ is piecewise constant on $Q$.

The last lemma relies on ideas due to J. Maly and M. Zeleny [8], and is also from [6]. The mapping $\mathbf{t}$ is defined using that a suitable game has a winning strategy.

Lemma 2.3. Let $B$ be a closed ball of $\mathbb{R}^{d}$. Then, there exists a map $\mathbf{t}$ : $B \rightarrow \mathbb{R}^{d}$ such that if a sequence $\left(\sigma_{n}\right) \in B$ satisfies $\left\langle\mathbf{t}\left(\sigma_{n}\right), \sigma_{n+1}-\sigma_{n}\right\rangle \geq 0$ for all $n$, then $\left(\sigma_{n}\right)$ converges.

\section{Almost classical solutions on open subsets of $\mathbb{R}^{d}$}

For a wide class of Hamilton-Jacobi equations, we give an existence theorem of almost classical solutions defined on the closure of on an open subset of $\mathbb{R}^{d}$, and satisfying an homogeneous Dirichlet condition.

Theorem 3.1. Let $\Omega$ be an open subset of $\mathbb{R}^{d}$ with $d \geq 2$, and $F: \mathbb{R} \times$ $\Omega \times \mathbb{R}^{d} \rightarrow \mathbb{R}$ be a continuous function. Suppose that the following conditions hold:

(A) $F(0, x, 0) \leq 0$, for every $x \in \Omega$; that is, the function $u_{0}$ identically equal to 0 is a classical subsolution of $F(u(x), x, \nabla u(x))=0$.

(B) For each compact subset $K \subset \Omega$, there exist $\alpha_{K}>0$ and $M_{K}>0$ such that for all $x \in K$, for all $u \in\left[0, \alpha_{K}\right]$ and for all $p \in \mathbb{R}^{d}$ satisfying $\|p\| \geq M_{K}$, we have $F(u, x, p)>0$. 
Then there exists a function $u \geq 0$ on $\bar{\Omega}$ which is an almost classical solution of $F(u(x), x, \nabla u(x))=0$, with Dirichlet condition $\left.u\right|_{\partial \Omega}=0$. Moreover, the extension $\widetilde{u}$ of $u$ to $\mathbb{R}^{d}$ satisfying $\widetilde{u}(x)=0$ if $x \notin \bar{\Omega}$ is differentiable at every point of $\mathbb{R}^{d}$.

The proof of Theorem 3.1 will be postponed until Section 4. Along this section, we will obtain several consequences of this result.

Remark 3.2. It will be useful to note that condition (B) in Theorem 3.1 is equivalent to condition(B') and also to condition (B") below:

(B') For each compact subset $K \subset \Omega$, there exists $\alpha_{K}>0$ such that the set

$$
B\left(K ; \alpha_{K}\right)=\left\{(u, x, p) \in\left[0, \alpha_{K}\right] \times K \times \mathbb{R}^{d}: F(x, u, p) \leq 0\right\}
$$

is compact in $\mathbb{R} \times \Omega \times \mathbb{R}^{d}$.

(B") For each $x_{0} \in \Omega$, there exist a compact neighborhood $V^{x_{0}}$ and $\alpha>0$, such that the set

$$
B\left(V^{x_{0}} ; \alpha\right)=\left\{(u, x, p) \in[0, \alpha] \times V^{x_{0}} \times \mathbb{R}^{d}: F(u, x, p) \leq 0\right\}
$$

is compact in $\mathbb{R} \times \Omega \times \mathbb{R}^{d}$.

We now consider the case of general Dirichlet conditions.

Corollary 3.3. Let $\Omega$ be an open subset of $\mathbb{R}^{d}$ with $d \geq 2$, and $F: \mathbb{R} \times$ $\Omega \times \mathbb{R}^{d} \rightarrow \mathbb{R}$ be a continuous function. Suppose that the following conditions hold:

(A) There exists a continuous function $u_{0}: \bar{\Omega} \rightarrow \mathbb{R}$, which is $\mathcal{C}^{1}$-smooth on $\Omega$ and such that $F\left(u_{0}(x), x, \nabla u_{0}(x)\right) \leq 0$, for every $x \in \Omega$.

(B) For each compact subset $K \subset \Omega$, there exist $M_{K}>0$ and $\alpha_{K}>0$ such that for all $x \in K$, for all $u \in\left[0, \alpha_{K}\right]$ and for all $p \in \mathbb{R}^{d}$ satisfying $\|p\| \geq M_{K}$, we have $F\left(u_{0}(x)+u, x, p\right)>0$.

Then there exists an almost classical solution $u$ of $F(u(x), x, \nabla u(x))=0$, with Dirichlet condition $\left.u\right|_{\partial \Omega}=u_{0}$. Moreover, if $u_{0}$ is $\mathcal{C}^{1}$-smooth on $\mathbb{R}^{d}$, the function $u$ can be extended to a differentiable function on $\mathbb{R}^{d}$.

Proof . Define $G(u, x, p)=F\left(u+u_{0}(x), x, p+\nabla u_{0}(x)\right)$. Conditions $(A)$ and $(B)$ of Theorem 3.1 are satisfied for $G$. Thus, there exists an almost classical solution $v$ of $G(v(x), x, \nabla v(x))=0$, with Dirichlet condition $\left.v\right|_{\partial \Omega}=0$, and furthermore $v$ can be extended to a differentiable function on $\mathbb{R}^{d}$. The function $u: \bar{\Omega} \rightarrow \mathbb{R}$ defined by $u(x)=u_{0}(x)+v(x)$ is then an almost classical solution of $F(u(x), x, \nabla u(x))=0$, with Dirichlet condition $\left.u\right|_{\partial \Omega}=u_{0}$. 
Notice that Theorem 1.2 is a straightforward consequence of Corollary 3.3. Another easy consequence of Corollary 3.3 is the following existence result of almost classical solutions for stationary Hamilton-Jacobi equations:

Corollary 3.4. Let $\Omega$ be an open subset of $\mathbb{R}^{d}$ with $d \geq 2$, and let $F$ : $\Omega \times \mathbb{R}^{d} \rightarrow \mathbb{R}$ be a continuous function. Suppose that the following conditions hold:

(A) There exists a continuous function $u_{0}: \bar{\Omega} \rightarrow \mathbb{R}$, which is $\mathcal{C}^{1}$-smooth on $\Omega$ and such that $u_{0}(x)+F\left(x, \nabla u_{0}(x)\right) \leq 0$, for every $x \in \Omega$.

(B) For each compact $K \subset \Omega$, there exists $M_{K}>0$ such that

$$
\inf \left\{u_{0}(x)+F(x, p) ; x \in K, p \in \mathbb{R}^{d},\|p\| \geq M_{K}\right\}>0
$$

Then there exists an almost classical solution of $u(x)+F(x, \nabla u(x))=0$, with Dirichlet condition $\left.u\right|_{\partial \Omega}=u_{0}$.

Next we give a further application of Theorem 3.1. We shall need the following notions. If $A$ is a subset of $\mathbb{R}^{d}$, we denote its complement by $A^{c}=\mathbb{R}^{d} \backslash A$. Let us recall the definition of the Hausdorff distance between closed sets of a metric space. If $X$ is a metric space, for each $A \subset X$ and $r>0$ we denote $B(A, r)=\{x \in X: \operatorname{dist}(x, A)<r\}$. We denote $\mathcal{C}(X)$ the set of all closed bounded subsets of $X$. If $C$ and $D$ are in $\mathcal{C}(X)$, the Hausdorff distance between them is

$$
d_{H}(C, D)=\inf \{r \in(0, \infty]: C \subset B(D, r) \text { and } D \subset B(C, r)\} .
$$

Theorem 3.5. Let $\Omega$ be an open subset of $\mathbb{R}^{d}$ with $d \geq 2$. For each $x \in \Omega$ let $U(x)$ be an open bounded subset of $\mathbb{R}^{d}$ containing 0 . Assume that the set-valued mapping $x \mapsto \partial U(x)$ from $\Omega$ into $\left(\mathcal{C}\left(\mathbb{R}^{d}\right), d_{H}\right)$ is continuous on $\Omega$. Then there exists a differentiable function $u: \mathbb{R}^{d} \rightarrow \mathbb{R}$ such that:

1. $\left.u\right|_{\Omega^{c}} \equiv 0$ and $\left.\nabla u\right|_{\Omega^{c}} \equiv 0$.

2. $\nabla u(x) \in \overline{U(x)}$ for every $x \in \mathbb{R}^{d}$.

3. $\nabla u(x) \in \partial U(x)$ for almost every $x \in \Omega$.

Proof. Consider the function $F: \mathbb{R} \times \mathbb{R}^{d} \times \mathbb{R}^{d} \rightarrow \mathbb{R}$ defined by $F(u, x, p)=$ $-\operatorname{dist}(p, \partial U(x))$ if $x \in U(x)$, and $F(u, x, p)=\operatorname{dist}(p, \partial U(x))$ otherwise. Since the mapping $x \mapsto \partial U(x)$ from $\Omega$ into $\left(\mathcal{C}\left(\mathbb{R}^{d}\right), d_{H}\right)$ is continuous on $\Omega$, is is easy to see that $F$ is continuous on $\mathbb{R} \times \mathbb{R}^{d} \times \mathbb{R}^{d}$. The function identically 
equal to 0 is a classical subsolution of $F(u, x, p)=0$. On the other hand, for each compact subset $K \subset \mathbb{R}^{d}$, there exists $R>0$ such that

$$
\bigcup_{x \in K} \partial U(x) \subset B(0, R)
$$

and therefore

$$
\bigcup_{x \in K} \overline{U(x)} \subset B(0, R)
$$

Hence for all $u \in \mathbb{R}$, for all $p \in \mathbb{R}^{d}$ satisfying $\|p\| \geq 2 R$ and for all $x \in K$, we have $F(u, x, p)>0$. The two hypothesis of Theorem 3.1 are then satisfied. The almost classical solution of $F(u, x, p)=0$ given by Theorem 3.1 satisfies the required properties.

\section{Proof of Theorem 3.1.}

In order to prove Theorem 3.1, we first consider the case of a cube, on which almost classical solutions will be obtained as the sum of a series of $\mathcal{C}^{\infty}$-smooth functions, and the general case will then follow easily.

Lemma 4.1. Assume that the hypotheses of Theorem 3.1 are satisfied, and let $C$ be a cube such that $\bar{C}$ is contained in $\Omega$. Then there exists a differentiable function $u_{C}: \mathbb{R}^{d} \rightarrow \mathbb{R}$ such that:

1. $u_{C} \geq 0$ and $u_{C}(x)=0$ for all $x$ which is not in the interior of $C$.

2. $F\left(u_{C}(x), x, \nabla u_{C}(x)\right) \leq 0$ for every $x \in C$.

3. $F\left(u_{C}(x), x, \nabla u_{C}(x)\right)=0$ for almost every $x \in C$.

Proof of Theorem 3.1. We first fix an increasing sequence $\left(K_{n}\right)_{n \geq 1}$ of compact subsets of $\Omega$ such that the union of all $K_{n}$ 's is equal to $\Omega$. We also assume that each $K_{n}$ is the closure of a finite union of cubes. By assumption $(B)$, for each $n \geq 1$ there exist $M_{n}>0$ and $\alpha_{n}>0$ such that, for all $x \in K_{n}$, for all $u \in\left[0, \alpha_{n}\right]$ and for all $p \in \mathbb{R}^{d}$ satisfying $\|p\| \geq M_{n}$, we have $F(u, x, p)>0$. We consider a decomposition

$$
\Omega=\bigcup_{j=1}^{\infty} C_{j},
$$

where $\left(C_{j}\right)_{j \geq 1}$ is a locally finite family of cubes such that:

(a) $C_{j} \cap C_{k}=\emptyset$ if $j \neq k$.

(b) for each $j$, there exists $n$ such that $C_{j} \subset \overline{K_{n} \backslash K_{n-1}}$. 
Refining if necessary this decomposition, we can also assume:

(c) $\operatorname{diam}\left(C_{j}\right) \leq \frac{1}{2^{n} M_{n}} d_{H}\left(K_{n}, \partial \Omega\right)$ whenever $C_{j} \subset \overline{K_{n} \backslash K_{n-1}}$.

By Lemma 4.1, for each $j \geq 1$ there exists a differentiable function $u_{j}$ : $\mathbb{R}^{d} \rightarrow \mathbb{R}$ such that:

1. $u_{j} \geq 0$ and $u_{j}(x)=0$ for all $x$ which is not in the interior of $C_{j}$.

2. $F\left(u_{j}(x), x, \nabla u_{j}(x)\right) \leq 0$ for every $x \in C_{j}$.

3. $F\left(u_{j}(x), x, \nabla u_{j}(x)\right)=0$ for almost every $x \in C_{j}$.

Then we define $u: \mathbb{R}^{d} \rightarrow \mathbb{R}$ by setting

$$
u=\sup _{j \geq 1} u_{j}
$$

By property (1) above, $u=u_{j}$ on each $C_{j}$. Then it is easy to see that $u$ is differentiable on $\Omega$, identically equal to 0 on $\mathbb{R}^{d} \backslash \Omega$, satisfies $F(u(x), x, \nabla u(x)) \leq$ 0 for every $x \in \Omega$ and $F(u(x), x, \nabla u(x))=0$ for almost every $x \in \Omega$. It remains to check that $u$ is differentiable at each point of $\partial \Omega$. Fix $n \geq 1$. We know that $u$ vanishes on the boundary of each cube $C_{j}$, so, by the mean value theorem,

$$
\sup \left\{u(x): x \in C_{j}\right\} \leq \sup \left\{\nabla u(x): x \in C_{j}\right\} \cdot \operatorname{diam}\left(C_{j}\right) .
$$

If $C_{j} \subset \overline{K_{n} \backslash K_{n-1}}$, then $\sup \left\{\nabla u(x): x \in C_{j}\right\} \leq M_{n}$. In that case, using (c), we obtain:

$$
\sup \left\{u(x): x \in C_{j}\right\} \leq \frac{1}{2^{n}} d_{H}\left(K_{n}, \partial \Omega\right) .
$$

So whenever $x \in K_{n} \backslash K_{n-1}$, we have that $0 \leq u(x) \leq \operatorname{dist}(x, \partial \Omega) / 2^{n}$. This implies that for each point $x \in \partial \Omega, u$ is differentiable at $x$ and $\nabla u(x)=0$.

Proof of Lemma 4.1. Observe that if $F(0, x, 0)=0$ for almost every $x \in C$, we can take $u_{C}=0$ and the above assertions are satisfied. From now on, we assume that

$$
\lambda_{d}(\{x \in C: F(0, x, 0)<0\})>0
$$

By assumption $(B)$, there exists $\alpha>0$ such that

$$
r:=\sup \{\|p\|: F(u, x, p) \leq 0 \text { for some } x \in \bar{C} \text { and } u \in[0, \alpha]\}
$$

is finite. We fix a map $\mathbf{t}: B(0,1+r) \rightarrow \mathbb{R}^{d}$ satisfying the conditions of Lemma 2.3. 
The function $u_{C}$ will be given by a series

$$
u_{C}=\sum_{n=1}^{\infty} u_{n}
$$

where each $u_{n}$ is a $\mathcal{C}^{\infty}$-smooth function on $\mathbb{R}^{d}$. For each $n$, we will write $\nabla u_{n}=v_{n}+w_{n}$, and we will denote

$$
U_{n}=\sum_{k=1}^{n} u_{k} \quad \text { and } \quad \sigma_{n}=\sum_{k=1}^{n} v_{k} .
$$

Construction of the functions $\boldsymbol{u}_{\boldsymbol{n}}$ : The functions $u_{n}$ will be constructed together with a sequence $\left(\mathcal{Q}_{n}\right)_{n \geq 0}$ of partitions of $C$ into cubes, where each $\mathcal{Q}_{n+1}$ is a refinement of $\mathcal{Q}_{n}$. We also fix a sequence $\left(\varepsilon_{k}\right)_{k \geq 1}$ of positive numbers, with $\left(\varepsilon_{k}\right) \downarrow 0$ and such that inf $\{F(0, x, 0): x \in C\}<-\varepsilon_{1}$ and $\varepsilon_{1}<1$, and we construct an increasing sequence of integers $\left(N_{k}\right)_{k \geq 0}$ with $N_{0}=0$. The following conditions will be proved by induction:

(0) There exists $x_{0} \in C$ such that, for each $n \geq 1, u_{n}\left(x_{0}\right)=0$ and $\nabla u_{n}\left(x_{0}\right)=0$.

(i) For each $n \geq 1, u_{n}$ and $v_{n}$ have their support included in the interior of $C, v_{n}$ is constant on each cube of $\mathcal{Q}_{n}$, and $\left\|w_{n}\right\|_{\infty} \leq 2^{-n}$.

(ii) For each $n \geq 1$ and $x \in C, F\left(U_{n}(x), x, \nabla U_{n}(x)\right) \leq 0$.

(iii) For each $n \geq 1$ and $x \in C$, we have

$$
\left\|\sigma_{n}(x)\right\| \leq 1+r \quad \text { and } \quad\left\langle\mathbf{t}\left(\sigma_{n}(x)\right), \sigma_{n+1}(x)-\sigma_{n}(x)\right\rangle=0,
$$

(iv) $\left\|u_{1}\right\|_{\infty} \leq \alpha / 2$, and, for each $n \geq 1$, we have

$$
0<\left\|u_{n+1}\right\|_{\infty} \leq 2^{-n}\left\|u_{n}\right\|_{\infty} \quad \text { and } \quad \operatorname{osc}\left(\nabla U_{n},\left\|u_{n+1}\right\|_{\infty}\right) \leq 1 / 2^{n}
$$

(v) For each $k \geq 1$ and each $N_{k-1}<n \leq N_{k}$, we have $\left\|v_{n}\right\|_{\infty} \leq \varepsilon_{k}$.

(vi) For each $k \geq 1$,

$$
\lambda_{d}\left\{x \in C: F\left(U_{N_{k}}(x), x, \nabla U_{N_{k}}(x)\right) \leq-\varepsilon_{k}\right\} \leq 2^{-k} \lambda_{d}(C) .
$$

Construction of $u_{1}$ : Fix a cube $Q_{0} \subset C$ with

$$
d_{H}\left(Q_{0}, \partial C\right)>0 \quad \text { and } \quad \sup \left\{F(0, x, 0): x \in Q_{0}\right\} \leq-\varepsilon_{1}
$$

This implies, using the uniform continuity of $F$ on compact sets, that there exists $0<\delta_{1} \leq \varepsilon_{1}$ such that, whenever $x \in Q_{0}, 0 \leq h \leq \delta_{1}$ and $\|q\| \leq 2 \delta_{1}$, then:

$$
F(h, x, q) \leq 0
$$


Choose $a=a\left(Q_{0}\right) \in \mathbb{R}^{d}$ such that $\left\|a\left(Q_{0}\right)\right\|=\delta_{1}$ and $\langle\mathbf{t}(0), a\rangle=0$ (this is possible since $d \geq 2$ ). Now applying Lemma 2.2 to the cube $Q_{0}$, we obtain a $C^{\infty}$ function $u_{1}$ on $\mathbb{R}^{d}$, and a cube partition $\mathcal{Q}_{1}$ of $C$ such that $Q_{0}$ is a union of some elements of $\mathcal{Q}_{1}$, such that:

- $u_{1}$ vanishes on a neighborhood of $\partial Q_{0}$ and outside of $Q_{0}$.

- $0<\left\|u_{1}\right\|_{\infty} \leq \min \left\{\delta_{1}, a / 2\right\}$.

- $\nabla u_{1}=v_{1}+w_{1}$, where $\left\|w_{1}\right\|_{\infty} \leq \min \left\{\delta_{1}, 1 / 2\right\}, v_{1}$ is constant on each cube of $\mathcal{Q}_{1}$, and $v_{1}\left(Q_{0}\right) \subset\left[-a\left(Q_{0}\right), a\left(Q_{0}\right)\right]$.

Fix $x_{0} \in \partial Q_{0}$ : we have $u_{1}\left(x_{0}\right)=0$ and $\nabla u_{1}\left(x_{0}\right)=0$, so condition (0) is satisfied. Conditions (i),(iii) and (v) are clearly satisfied, and (ii) follows from (4.1). So we can start the induction.

Inductive step: Fix $k \geq 1$, assume that $N_{k-1}$ has been defined, and for some $n \geq N_{k-1}$ the partition $\mathcal{Q}_{n}$ and the function $u_{n}$ have been constructed.

First, there exists $0<\delta_{k} \leq \varepsilon_{k}$ such that whenever $x \in \bar{C}, u \in[0, \alpha]$, $p \in B(0,1+r), 0 \leq h \leq \delta_{k}$ and $\|q\| \leq 2 \delta_{k}$, then

$$
F(u, x, p) \leq-\varepsilon_{k} / 2 \quad \Longrightarrow \quad F(u+h, x, p+q) \leq 0
$$

Next, choose a cube partition $\widehat{\mathcal{Q}}_{n}$ of $C$ refining $\mathcal{Q}_{n}$ such that:

○ If we denote $\widehat{\mathcal{R}}_{n}$ the family of all cubes $Q \in \widehat{\mathcal{Q}}_{n}$ such that $d_{H}(Q, \partial C)>0$, and $K_{n}=\cup\left\{Q: Q \in \widehat{\mathcal{R}}_{n}\right\}$, we have that

$$
\lambda_{d}\left(C \backslash K_{n}\right)<2^{-(k+1)} \lambda_{d}(C) .
$$

○ For all $Q \in \widehat{\mathcal{R}}_{n}$ and every $x, y \in Q$, we have

$$
\left|F\left(U_{n}(x), x, \nabla U_{n}(x)\right)-F\left(U_{n}(y), y, \nabla U_{n}(y)\right)\right|<\varepsilon_{k} / 2 .
$$

The second condition above can be obtained using the uniform continuity of the mapping $x \mapsto F\left(U_{n}(x), x, \nabla U_{n}(x)\right)$ on the compact set $\bar{C}$.

Now, each cube $Q$ of $\widehat{\mathcal{Q}}_{n}$ is contained in a cube $Q^{\prime}$ of $\mathcal{Q}_{n}$ and by (i), $\sigma_{n}$ is constant on $Q^{\prime}$. We denote by $\sigma_{n}(Q)$ the constant value of $\left.\sigma_{n}\right|_{Q^{\prime}}$. Choose $a=a(Q) \in \mathbb{R}^{d}$ such that $\|a(Q)\|=\delta_{k}$ and $\left\langle\mathbf{t}\left(\sigma_{n}(Q)\right), a\right\rangle=0$. Now applying Lemma 2.2, for each cube $Q \in \widehat{\mathcal{R}}_{n}$ we obtain a $C^{\infty}$ function $u_{Q}$ on $\mathbb{R}^{d}$, and a cube partition $\mathcal{Q}_{n+1}$ of $Q_{0}$ which is a refinement of $\widehat{\mathcal{Q}}_{n}$ (and therefore of $\mathcal{Q}_{n}$ ), such that:

(a) $u_{Q}$ vanishes on a neighborhood of $\partial Q$.

(b) $0<\left\|u_{Q}\right\|_{\infty} \leq \min \left\{2^{-n}\left\|u_{n}\right\|_{\infty}, \delta_{k}\right\}$ and $\operatorname{osc}\left(\nabla U_{n},\left\|u_{Q}\right\|_{\infty}\right)<1 / 2^{n}$. 
(c) $\lambda_{d}\left\{x \in Q: \nabla u_{Q}(x)= \pm a(Q)\right\} \geq\left(1-2^{-k}\right) \lambda_{d}(Q)$.

(d) $\nabla u_{Q}=v_{Q}+w_{Q}$, where $\left\|w_{Q}\right\|_{\infty} \leq \min \left\{\delta_{k}, 1 / 2^{n+2}\right\}, v_{Q}$ is constant on each cube of $\mathcal{Q}_{n+1}$, and $v_{Q}(Q) \subset[-a(Q), a(Q)]$. In particular, we have $\left\|v_{Q}\right\|_{\infty} \leq\|a(Q)\|=\delta_{k} \leq \varepsilon_{k}$ and $\left\|\nabla u_{Q}\right\|_{\infty} \leq 2 \delta_{k}$.

Next we define the function $u_{n+1}$ on $\mathbb{R}^{d}$. We first choose for each $Q \in \widehat{\mathcal{R}}_{n}$ a point $x_{Q}$ in the closure of $Q$ such that

$$
F\left(U_{n}\left(x_{Q}\right), x_{Q}, \nabla U_{n}\left(x_{Q}\right)\right)=\inf \left\{F\left(U_{n}(x), x, \nabla U_{n}(x)\right): x \in Q\right\}
$$

We define $u_{n+1}$ on each cube of $\widehat{\mathcal{R}}_{n}$ in the following way:

1. If $F\left(U_{n}\left(x_{Q}\right), x_{Q}, \nabla U_{n}\left(x_{Q}\right)\right)>-\varepsilon_{k}$, we set $u_{n+1}=0$ and $v_{n+1}=$ $w_{n+1}=0$ on $Q$.

2. If $F\left(U_{n}\left(x_{Q}\right), x_{Q}, \nabla U_{n}\left(x_{Q}\right)\right) \leq-\varepsilon_{k}$, we set $u_{n+1}=u_{Q}$ on $Q$. In this case, $v_{n+1}=v_{Q}, w_{n+1}=w_{Q}$, and we have

$$
\lambda_{d}\left\{x \in Q:\left\|\nabla u_{n+1}(x)\right\|=\delta_{k}\right\} \geq\left(1-2^{-k}\right) \cdot \lambda_{d}(Q) .
$$

3. Finally, on $\left(K_{n}\right)^{c}$, we set $u_{n+1}=0$, and $v_{n+1}=w_{n+1}=0$.

In this way we obtain that $u_{n+1}$ is a $C^{\infty}$ function on $\mathbb{R}^{d}$, which vanish on a neighborhood of $\partial Q$ for every $Q \in \widehat{\mathcal{R}}_{n}$.

Next we are going to check conditions $(0)$ to (vi) for $n+1$. Since $\widehat{\mathcal{Q}}_{n}$ is a refinement of $\mathcal{Q}_{1}$ and $x_{0} \in \partial Q_{0} \in \mathcal{Q}_{1}$, there exists $Q \in \widehat{\mathcal{Q}}_{n}$ such that $x_{0} \in \partial Q$, so $u_{n+1}\left(x_{0}\right)=0$ and $\nabla u_{n+1}\left(x_{0}\right)=0$. This proves condition (0). Condition (i) is clearly satisfied. In order to prove condition (ii), fix $x \in \Omega$. By induction hypothesis, $F\left(U_{n}(x), x, \nabla U_{n}(x)\right) \leq 0$. Let us prove that $F\left(U_{n+1}(x), x, \nabla U_{n+1}(x)\right) \leq 0$ :

- If $x \in\left(K_{n}\right)^{c}$, then $u_{n+1}=0$ on a neighbourhood of $x$ and $\nabla U_{n+1}(x)=$ $\nabla U_{n}(x)$, so $F\left(U_{n+1}(x), x, \nabla U_{n+1}(x)\right)=F\left(U_{n}(x), x, \nabla U_{n}(x)\right) \leq 0$.

- If $x \in Q \in \widehat{\mathcal{R}}_{n}$ with $F\left(U_{n}\left(x_{Q}\right), x_{Q}, \nabla U_{n}\left(x_{Q}\right)\right)>-\varepsilon_{k}$, then $u_{n+1}=0$ on a neighborhood of $Q$ and

$$
F\left(U_{n+1}\left(x_{Q}\right), x_{Q}, \nabla U_{n+1}\left(x_{Q}\right)\right)=F\left(U_{n}\left(x_{Q}\right), x_{Q}, \nabla U_{n}\left(x_{Q}\right)\right) \leq 0 .
$$

- Finally, if $x \in Q \in \widehat{\mathcal{R}}_{n}$ with $F\left(U_{n}\left(x_{Q}\right), x_{Q}, \nabla U_{n}\left(x_{Q}\right)\right) \leq-\varepsilon_{k}$, by 4 .4, we have for all $x \in Q, F\left(U_{n}(x), x, \nabla U_{n}(x)\right) \leq-\varepsilon_{k} / 2$. Since

$$
\left|U_{n}(x)\right| \leq \sum_{k=1}^{\infty}\left|u_{k}(x)\right| \leq \alpha,
$$


from the definition of $r$ and the fact that $F\left(U_{n}(x), x, \nabla U_{n}(x)\right) \leq 0$, it follows that $\left\|\nabla U_{n}(x)\right\| \leq r$. Since we have also $\left\|u_{n+1}\right\|_{\infty} \leq \delta_{k}$ and $\left\|\nabla u_{n+1}\right\|_{\infty} \leq 2 \delta_{k}$, we deduce from 4.2 that

$$
F\left(U_{n+1}(x), x, \nabla U_{n+1}(x)\right) \leq 0
$$

In order to prove (iii), fix $x \in C$. First, $F\left(U_{n+1}(x), x, \nabla U_{n+1}(x)\right) \leq 0$, so $\left\|\nabla U_{n+1}(x)\right\| \leq r$, and

$$
\left\|\nabla U_{n+1}(x)-\sigma_{n+1}(x)\right\| \leq \sum_{k=1}^{n+1}\left\|w_{k}(x)\right\| \leq 1 .
$$

Therefore $\left\|\sigma_{n}(x)\right\| \leq 1+r$. Then, if $v_{n+1}(x)=0$, we have $\sigma_{n+1}(x)-\sigma_{n}(x)=0$, so $\left\langle\mathbf{t}\left(\sigma_{n}(x)\right), \sigma_{n+1}(x)-\sigma_{n}(x)\right\rangle=0$. On the other hand, if $v_{n+1}(x) \neq 0$, then $x \in Q$ for some $Q \in \widehat{\mathcal{R}}_{n}$, and $v_{n+1}=v_{Q}$. In this case $\sigma_{n}(x)=\sigma_{n}(Q)$, and $v_{n+1}(x) \in[-a(Q), a(Q)]$. Thus $v_{n+1}(x)=\sigma_{n+1}(x)-\sigma_{n}(x)$ is proportional to $a(Q)$, and therefore orthogonal to $\mathbf{t}\left(\sigma_{n}(Q)\right)$, and the condition $\left\langle\mathbf{t}\left(\sigma_{n}(x)\right), \sigma_{n+1}(x)-\sigma_{n}(x)\right\rangle=0$ is again satisfied.

Now we are going to see that $u_{n+1} \neq 0$. Indeed, if $Q \in \widehat{\mathcal{Q}}_{n}$ is such that $Q \subset Q_{0}$ and $x_{0} \in \partial Q$, then $d_{H}(Q, \partial C) \geq d_{H}\left(Q_{0}, \partial C\right)>0$, so $Q \in \widehat{\mathcal{R}}_{n}$. Since $U_{n}\left(x_{0}\right)=0$ and $\nabla U_{n}\left(x_{0}\right)=0$, we have

$$
F\left(U_{n}\left(x_{Q}\right), x_{Q}, \nabla U_{n}\left(x_{Q}\right)\right) \leq F\left(U_{n}\left(x_{0}\right), x_{0}, \nabla U_{n}\left(x_{0}\right)\right) \leq-\varepsilon_{1} \leq-\varepsilon_{k},
$$

and therefore $u_{n+1}=u_{Q} \neq 0$ on $Q$. Condition (iv) follows now from (b). Next, condition (v) also holds, although we still have to define the integer $N_{k}$.

Finally, let us prove that (vi) is satisfied. Suppose, to the contrary, that for every $n>N_{k-1}$ we have:

$$
\lambda_{d}\left\{x \in C: F\left(U_{n}(x), x, \nabla U_{n}(x)\right) \leq-\varepsilon_{k}\right\}>2^{-k} \cdot \lambda_{d}(C) .
$$

By (4.3), we obtain that

$$
\lambda_{d}\left\{x \in K_{n}: F\left(U_{n}(x), x, \nabla U_{n}(x)\right) \leq-\varepsilon_{k}\right\}>2^{-(k+1)} \cdot \lambda_{d}\left(K_{n}\right) .
$$

Suppose now that

$$
F\left(U_{n}\left(x_{Q}\right), x_{Q}, \nabla U_{n}\left(x_{Q}\right)\right) \leq-\varepsilon_{k} .
$$

As we have noticed, in this case

$$
\begin{aligned}
& \lambda_{d}\left\{x \in Q:\left\|\nabla U_{n+1}(x)-\nabla U_{n}(x)\right\| \geq \delta_{k}\right\} \geq \\
& \quad \geq \lambda_{d}\left\{x \in Q:\left\|\nabla u_{n+1}(x)\right\|=\delta_{k}\right\} \geq\left(1-2^{-k}\right) \cdot \lambda_{d}(Q) .
\end{aligned}
$$


Now the proportion of cubes $Q$ in $\widehat{\mathcal{R}}_{n}$ satisfying this has to be at least $2^{-(k+1)}$. Therefore

$$
\lambda_{d}\left\{x \in K_{n}:\left\|\nabla U_{n+1}(x)-\nabla U_{n}(x)\right\| \geq \delta_{k}\right\} \geq\left(1-2^{-k}\right) 2^{-(k+1)}>0 .
$$

This will be a contradiction with Lemma 2.3, since we are going to prove that the sequence $\left(\nabla U_{n}\right)_{n \geq 1}$ is pointwise convergent. Indeed, for each $x \in \mathbb{R}^{d}$, it follows from (i) that the sequence $\left(\sum_{k=1}^{n} w_{k}(x)\right)_{n>1}$ converges, and from (iii) and Lemma 2.3 that the sequence $\left(\sigma_{n}(x)\right)_{n \geq 1}$ converges. Since

$$
\nabla U_{n}(x)=\sigma_{n}(x)+\sum_{k=1}^{n} w_{k}(x)
$$

we have that the sequence $\left(\nabla U_{n}(x)\right)_{n>1}$ is convergent. This contradiction shows that there exists an integer $N_{k}>N_{k+1}$ satisfying (vi). This concludes the inductive step.

The function $\boldsymbol{u}_{C}$ : We now define

$$
u_{C}=\sum_{n=1}^{\infty} u_{n}
$$

By (vi) the series is uniformly convergent on $\mathbb{R}^{d}$, so that $u: \mathbb{R}^{d} \rightarrow \mathbb{R}$ is a continuous function, and it is clear that $u_{C}$ vanishes outside $C$. In order to see that $u_{C}$ is differentiable on $\mathbb{R}^{d}$, we check the conditions of Lemma 2.1. For each $n \geq 1$ let $k_{n}$ be an integer with $N_{k_{n}-1}<n \leq N_{k_{n}}$. From (i) and (v), we have that

- $\left\|\nabla u_{n}\right\|_{\infty} \leq\left\|v_{n}\right\|_{\infty}+\left\|w_{n}\right\|_{\infty} \leq \varepsilon_{k_{n}}+2^{-n} \rightarrow 0$

and from (iv), we obtain:

- $\left\|\nabla u_{n+1}\right\|_{\infty}=o\left(\left\|\nabla u_{n}\right\|_{\infty}\right)$,

- $\operatorname{osc}\left(\nabla U_{n},\left\|\nabla u_{n}\right\|_{\infty}\right) \leq 2^{-n} \rightarrow 0$.

Moreover, applying as before Lemma 2.3 , the sequence $\left(\nabla U_{n}\right)_{n \geq 1}$ is pointwise convergent, that is,

$$
\sum_{n=1}^{\infty} \nabla u(x)
$$


is convergent for every $x \in \mathbb{R}^{d}$. Applying Lemma 2.1, we obtain that $u_{C}$ is everywhere differentiable and $\nabla u_{C}$ is the pointwise limit of

$$
\sum_{k=1}^{n} u_{k} .
$$

Now (ii) implies that $F\left(u_{C}(x), x, \nabla u_{C}(x)\right) \leq 0$ for every $x \in \Omega$. Finally, let us prove that

$$
F\left(u_{C}(x), x, \nabla u_{C}(x)\right)=0
$$

almost everywhere on $C$. Consider $x \in C$ such that $F\left(u_{C}(x), x, \nabla u_{C}(x)\right)<0$. Taking into account that $\nabla u_{C}(x)=\lim _{k} \nabla U_{N_{k}}(x)$, we can find some integer $k_{0}$ such that

$$
F\left(U_{N_{k}}(x), x, \nabla U_{N_{k}}(x)\right)<-\varepsilon_{k_{0}} \leq-\varepsilon_{k},
$$

for every $k \geq k_{0}$. Therefore the set $\left\{x \in C: F\left(u_{C}(x), x, \nabla u_{C}(x)\right)<0\right\}$ is contained in the set

$$
\limsup _{k}\left\{x \in C: F\left(U_{N_{k}}(x), x, \nabla U_{N_{k}}(x)\right)<-\varepsilon_{k}\right\} .
$$

Since

$$
\lambda_{d}\left\{x \in C: F\left(U_{N_{k}}(x), x, \nabla U_{N_{k}}(x)\right)<-\varepsilon_{k}\right\} \leq 2^{-k} \lambda_{d}(C) \rightarrow 0,
$$

by Borel-Cantelli lemma $\lambda_{d}\left(\left\{x \in C: F\left(u_{C}(x), x, \nabla u_{C}(x)\right)<0\right\}\right)=0$. That is, $F\left(u_{C}(x), x, \nabla u_{C}(x)\right)=0$ for almost every $x \in C$.

\section{Almost classical solutions on Riemannian manifolds}

In order to obtain our results for smooth manifolds, we will use the concept of triangulation, as it is given by Whitney in [11] (see also [3]). In what follows we assume that every smooth manifold is Hausdorff and second countable. If $M$ is a smooth $d$-dimensional manifold, a triangulation of $M$ is a pair $(K, \pi)$, where $K$ is a simplicial complex and $\pi: K \rightarrow M$ is a homeomorphism, such that for each $d$-dimensional simplex $S$ of $K$ there exists a local chart $(W, \varphi)$ of $M$, where $W$ is a neighborhood of $\pi(S)$ and $\varphi \circ \pi$ is affine on $S$. According to Whitney [11], every smooth manifold admits a triangulation.

Our first result is an extension of Theorem 3.1 to the setting of smooth manifolds. The Riemannian structure is not needed here. We consider an open subset $\Omega$ of the manifold $M$ and we denote $T^{*} \Omega$ the corresponding cotangent bundle. Then we consider equations of the form $F(u(x), x, d u(x))=0$, where $F: \mathbb{R} \times T^{*} \Omega \rightarrow \mathbb{R}$ is a continuous function. We obtain the following: 
Theorem 5.1. Let $M$ be a smooth manifold of dimension $d \geq 2$, consider an open subset $\Omega$ of $M$, and let $F: \mathbb{R} \times T^{*} \Omega \rightarrow \mathbb{R}$ be a continuous function. Suppose that the following conditions hold:

(A) There exists a $\mathcal{C}^{1}$ function $u_{0}: M \rightarrow \mathbb{R}$ such that $F\left(u_{0}(x), x, d u_{0}(x)\right) \leq 0$, for every $x \in \Omega$.

(B) For each $x_{0} \in \Omega$, there exist a compact neighborhood $V^{x_{0}}$ in $\Omega$ and $\alpha>0$, such that the set $B\left(V^{x_{0}} ; \alpha\right)$ is compact in $\mathbb{R} \times T^{*} M$, where

$$
\begin{gathered}
B\left(V^{x_{0}} ; \alpha\right)=\left\{(u, x, \xi) \in \mathbb{R} \times T^{*} M: u \in[0, \alpha] ; x \in V^{x_{0}} ;\right. \\
\left.F\left(u+u_{0}(x), x, \xi\right) \leq 0\right\} .
\end{gathered}
$$

Then there exists a differentiable function $u: M \rightarrow \mathbb{R}$ such that:

1. $u \geq u_{0}$ on $M, u=u_{0}$ on $\Omega^{c}$ and $d u=d u_{0}$ on $\Omega^{c}$.

2. $F(u(x), x, d u(x)) \leq 0$ for every $x \in \Omega$.

3. $F(u(x), x, d u(x))=0$ for almost every $x \in \Omega$.

Proof. We will consider two cases.

First Case: Suppose first that $u_{0} \equiv 0$ on $M$. Let $(K, \pi)$ be a triangulation of $M$, where $K$ is a simplicial complex and $\pi: K \rightarrow M$ is a homeomorphism, and consider the family $\left\{S_{i}\right\}_{i \in I}$ of all $d$-dimensional simplices of $K$. For each $i \in I$, denote $T_{i}=\pi\left(S_{i}\right)$. Then

$$
M=\bigcup_{i \in I} T_{i}
$$

each $\partial T_{i}$ has measure zero in $M$, and $\operatorname{int}\left(T_{i}\right) \cap \operatorname{int}\left(T_{j}\right)=\emptyset$ if $i \neq j$. Since $M$ is locally compact and $\pi$ is a homeomorphism, we have that the simplicial complex $K$ is locally compact, and therefore locally finite. Thus the family $\left\{T_{i}\right\}_{i \in I}$ is locally finite. Since $M$ is also $\sigma$-compact, we obtain that the index set $I$ is countable. For each $i \in I$, denote $\Omega_{i}=\Omega \cap \operatorname{int}\left(T_{i}\right)$. Then the set $\Omega \backslash\left(\cup_{i \in I} \Omega_{i}\right)$ has measure zero in $M$.

For each $i \in I$ there is a chart $\left(W_{i}, \varphi_{i}\right)$ in $M$ with $T_{i} \subset W_{i}$. Associated to this chart there is a natural diffeomorphism

$$
\Phi_{i}: \mathbb{R} \times T^{*} W_{i} \rightarrow \mathbb{R} \times \varphi_{i}\left(W_{i}\right) \times \mathbb{R}^{d}
$$

of the form $\Phi_{i}(u, x, \xi)=\left(u, \varphi_{i}(x), h_{i}(x, \xi)\right)$, where $h_{i}(x, \xi) \in \mathbb{R}^{d}$ satisfies that, for every $p \in \mathbb{R}^{d}$ :

$$
\left\langle h_{i}(x, \xi), p\right\rangle=\xi \circ d \varphi_{i}(x)^{-1}(p) .
$$


If $\varphi_{i}\left(\Omega_{i}\right) \neq \emptyset$, consider the function $G_{i}=F \circ \Phi_{i}^{-1}: \mathbb{R} \times \varphi_{i}\left(W_{i}\right) \times \mathbb{R}^{d} \rightarrow \mathbb{R}$. In order to apply Theorem 3.1 to the function $G_{i}$ note that the following conditions hold:

(A) $G_{i}(0, z, 0)=F\left(0, \varphi_{i}^{-1}(z), 0\right) \leq 0$, for each $z \in \varphi_{i}\left(\Omega_{i}\right)$.

(B) For each compact subset $H$ of $\varphi_{i}\left(\Omega_{i}\right)$, there exists $\alpha_{H}>0$ such that the set

$$
B\left(H ; \alpha_{H}\right)=\left\{(u, z, p) \in\left[0, \alpha_{H}\right] \times H \times \mathbb{R}^{d}: G_{i}(u, z, p) \leq 0\right\}
$$

is compact in $\mathbb{R} \times \varphi_{i}\left(W_{i}\right) \times \mathbb{R}^{d}$.

Therefore, taking into account Remark 3.2, we obtain that there exists a differentiable function $v_{i}: \varphi_{i}\left(W_{i}\right) \rightarrow \mathbb{R}$ such that:

1. $\left.v_{i}\right|_{\varphi_{i}\left(\Omega_{i}\right)^{c}} \equiv 0$ and $\left.\nabla v_{i}\right|_{\varphi_{i}\left(\Omega_{i}\right)^{c}} \equiv 0$;

2. $G_{i}\left(v_{i}(z), z, \nabla v_{i}(z)\right) \leq 0$ for every $z \in \varphi_{i}\left(\Omega_{i}\right)$.

3. $G_{i}\left(v_{i}(z), z, \nabla v_{i}(z)\right)=0$ for almost every $z \in \varphi_{i}\left(\Omega_{i}\right)$.

Then the function $u_{i}=v_{i} \circ \varphi_{i}: W_{i} \rightarrow \mathbb{R}$ is differentiable on $W_{i}$, and for each $x \in W_{i}$ we have that

$$
\begin{aligned}
F\left(u_{i}(x), x, d u_{i}(x)\right) & =F\left(u_{i}(x), x, d v_{i}\left(\varphi_{i}(x)\right) \circ d \varphi_{i}(x)\right) \\
& =F\left(\Phi_{i}^{-1}\left(v_{i}\left(\varphi_{i}(x)\right), \varphi_{i}(x), \nabla v_{i}\left(\varphi_{i}(x)\right)\right)\right) \\
& =G_{i}\left(v_{i}\left(\varphi_{i}(x)\right), \varphi_{i}(x), \nabla v_{i}\left(\varphi_{i}(x)\right)\right) .
\end{aligned}
$$

As a consequence, we obtain that

1. $\left.u_{i}\right|_{\Omega_{i}^{c}} \equiv 0$ and $\left.\nabla u_{i}\right|_{\Omega_{i}^{c}} \equiv 0$.

2. $F\left(u_{i}(x), x, d u_{i}(x)\right) \leq 0$ for every $x \in \Omega_{i}$.

3. $F\left(u_{i}(x), x, d u_{i}(x)\right)=0$ for almost every $x \in \Omega_{i}$.

On the other hand, if If $\varphi_{i}\left(\Omega_{i}\right)=\emptyset$, we set $u_{i}=0$. Now we define $u: M \rightarrow \mathbb{R}$ by setting $u=u_{i}$ on each $T_{i}$. Then $u$ is well-defined, since $\partial T_{i} \subset \Omega_{i}^{c}$ for each $i \in I$. Taking into account that the family $\left\{T_{i}\right\}_{i \in I}$ is locally finite, we see that $u$ is differentiable on $M$, and it satisfies the required conditions.

General Case: In general, consider the continuous function $G: \mathbb{R} \times T^{*} \Omega \rightarrow$ $\mathbb{R}$ defined by:

$$
G(u, x, \eta)=F\left(u+u_{0}(x), x, \eta+d u_{0}(x)\right) .
$$


We know that, or each $x_{0} \in \Omega$, there exist a compact neighborhood $V^{x_{0}}$ in $\Omega$ and $\alpha>0$, such that

$$
B_{F}\left(V^{x_{0}} ; \alpha\right)=\left\{(u, x, \xi) \in[0, \alpha] \times T^{*} \Omega: x \in V^{x_{0}} ; F\left(u+u_{0}(x), x, \xi\right) \leq 0\right\}
$$

is compact in $\mathbb{R} \times T^{*} \Omega$. Since the mapping $\tau: \mathbb{R} \times T^{*} \Omega \rightarrow \mathbb{R} \times T^{*} \Omega$ given by

$$
\tau(u, x, \xi)=\left(u, x, \xi-d u_{0}(x)\right)
$$

is continuous, we have that the set

$$
\begin{aligned}
B_{G}\left(V^{x_{0}} ; \alpha\right) & =\left\{(u, x, \eta) \in[0, \alpha] \times T^{*} \Omega: x \in V^{x_{0}} ; G\left(u+u_{0}(x), x, \eta\right) \leq 0\right\} \\
& =\tau\left(B_{F}\left(V^{x_{0}} ; \alpha\right)\right)
\end{aligned}
$$

is compact in $\mathbb{R} \times T^{*} \Omega$. Thus by the first case we obtain that there exists a differentiable function $v: M \rightarrow \mathbb{R}$ such that:

1. $\left.v\right|_{\Omega_{0}^{c}} \equiv 0$ and $\left.d v\right|_{\Omega_{0}^{c}} \equiv 0$.

2. $F(v(x), x, d v(x)) \leq 0$ for every $x \in \Omega$.

3. $F(v(x), x, d v(x))=0$ for almost every $x \in \Omega$.

Now it is easy to see that the function $u=u_{0}+v$ satisfies the required properties.

Our next Corollary, which is analogous to Corollary 3.4, is an easy consequence of Theorem 5.1.

Corollary 5.2. Let $M$ be a smooth manifold of dimension $d \geq 2$, consider an open subset $\Omega$ of $M$, and let $F: T^{*} \Omega \rightarrow \mathbb{R}$ be a continuous function. Suppose that the following conditions hold:

(A) There exists a $\mathcal{C}^{1}$ function $u_{0}: M \rightarrow \mathbb{R}$ such that $u_{0}(x)+F\left(x, d u_{0}(x)\right) \leq 0$, for every $x \in \Omega$.

(B) For each $x_{0} \in \Omega$, there exists a compact neighborhood $V^{x_{0}}$ in $\Omega$ such that the set $B\left(V^{x_{0}}\right)=\left\{(x, \xi) \in T^{*} M: x \in V^{x_{0}} ; u_{0}(x)+F(x, \xi) \leq 0\right\}$ is compact in $T^{*} M$.

Then there exists a differentiable function $u: M \rightarrow \mathbb{R}$ such that:

1. $u \geq u_{0}$ on $M, u=u_{0}$ on $\Omega^{c}$ and $d u=d u_{0}$ on $\Omega^{c}$.

2. $u(x)+F(x, d u(x)) \leq 0$ for every $x \in \Omega$.

3. $u(x)+F(x, d u(x))=0$ for almost every $x \in \Omega$. 
Also as a consequence of Theorem 5.1 we obtain the following result, which extends Theorem 1.3:

Theorem 5.3. Let $M$ be a smooth manifold of dimension $d \geq 2$, consider an open subset $\Omega$ of $M$, and let $F: T^{*} \Omega \rightarrow \mathbb{R}$ be a $\mathcal{C}^{1}$-smooth function. Suppose that the following conditions hold:

(A) There exists a $\mathcal{C}^{1}$-smooth function $u_{0}: M \rightarrow \mathbb{R}$ such that $F\left(x, d u_{0}(x)\right) \leq$ 0 , for every $x \in \Omega$.

(B) For each $x \in \Omega$, the set $B(x)=\left\{\xi \in T_{x}^{*} M: F(x, \xi) \leq 0\right\}$ is compact, the set $S(x)=\left\{\xi \in T_{x}^{*} M: F(x, \xi)=0\right\}$ is connected, and the function $F(x, \cdot)$ has maximal rank on the set $S(x)$.

Then there exists a differentiable function $u: M \rightarrow \mathbb{R}$ such that:

1. $u \geq u_{0}$ on $M, u=u_{0}$ on $\Omega^{c}$ and $d u=d u_{0}$ on $\Omega^{c}$.

2. $F(x, d u(x)) \leq 0$ for every $x \in \Omega$.

3. $F(x, d u(x))=0$ for almost every $x \in \Omega$.

Proof. We are going to see that the conditions of Theorem 5.1 are satisfied. Fix $x_{0} \in \Omega$, and consider a chart $(W, \varphi)$ in $M$ with $x_{0} \in W$. Associated to this chart, consider as before the natural diffeomorphism

$$
\Phi: T^{*} W \rightarrow \varphi(W) \times \mathbb{R}^{d}
$$

of the form $\Phi(x, \xi)=(\varphi(x), h(x, \xi))$, where $h(x, \xi) \in \mathbb{R}^{d}$ satisfies that, for every $p \in \mathbb{R}^{d}$ :

$$
\langle h(x, \xi), p\rangle=\xi \circ d \varphi(x)^{-1}(p) .
$$

Denote $z_{0}=\varphi\left(x_{0}\right)$. We take into account that $\Phi\left(S\left(x_{0}\right)\right)$ is compact, and that $F \circ \Phi^{-1}$ has maximal rank on $\left\{z_{0}\right\} \times \Phi\left(S\left(x_{0}\right)\right)$, and we apply the Implicit Function Theorem. Then we can find a neighborhood $U^{z_{0}}$ contained in $\varphi(W)$ and a finite family $V_{1}, \ldots V_{m}$ of open subsets of $\mathbb{R}^{d}$ with compact closure such that $\Phi\left(S\left(x_{0}\right)\right) \subset V_{1} \cup \cdots \cup V_{m}$ and, for each $j=1, \ldots, m$, the set of points $(z, p) \in U^{z_{0}} \times V_{j}$ satisfying $F \circ \Phi^{-1}(z, p)=0$ coincides, up to a permutation in the coordinates of $p$, with the graph of a $\mathcal{C}^{1}$-smooth mapping $g_{j}: U^{z_{0}} \times W_{j} \rightarrow \mathbb{R}$, where $W_{j}$ is an open subset of $\mathbb{R}^{d-1}$.

We claim that there exists a compact neighborhood $V^{x_{0}}$ such that, for every $x \in V^{x_{0}}$, we have that $\Phi(S(x)) \subset V_{1} \cup \cdots \cup V_{m}$. Indeed, if this is not the case, there exist a sequence $\left(z_{n}\right)_{n} \subset U^{z_{0}}$ converging to $z_{0}$ and a sequence $\left(p_{n}\right)_{n} \subset\left(V_{1} \cup \cdots \cup V_{m}\right)^{c}$ such that $F \circ \Phi^{-1}\left(z_{n}, p_{n}\right)=0$ for every $n$. Since each 
$S\left(x_{n}\right)$ is connected and $\Phi\left(S\left(x_{n}\right)\right) \cap\left(V_{1} \cup \cdots \cup V_{m}\right) \neq \emptyset$ for every $n$, we can assume that, in fact, $\left(p_{n}\right)_{n} \subset \partial\left(V_{1} \cup \cdots \cup V_{m}\right)$, which is a compact set. Then, taking a subsequence, we can assume that $\left(p_{n}\right)_{n}$ is convergent to some point $p_{0} \in \partial\left(V_{1} \cup \cdots \cup V_{m}\right)$. Now $F \circ \Phi^{-1}\left(z_{0}, p_{0}\right)=\lim _{n} F \circ \Phi^{-1}\left(z_{n}, p_{n}\right)=0$, that is, $p_{0} \in \Phi\left(S\left(x_{0}\right)\right)$, and this contradicts the fact that $\Phi\left(S\left(x_{0}\right)\right) \subset V_{1} \cup \cdots \cup V_{m}$. Then there exists $R>0$ such that $\Phi(S(x)) \subset B(0, R)$ for every $x \in V^{x_{0}}$. Since $\Phi(S(x))$ is the boundary of $\Phi(B(x))$ we have that in fact $\Phi(B(x)) \subset$ $B(0, R)$ for every $x \in V^{x_{0}}$. Thus the set

$$
B\left(V^{x_{0}}\right)=\left\{(x, \xi) \in T^{*} \Omega: x \in V^{x_{0}} ; F(x, \xi) \leq 0\right\}
$$

is compact in $T^{*} \Omega$, and the requirements of Theorem 5.1 are satisfied.

In our next result we consider a Riemannian manifold $(M, g)$. As we mentioned before, if $u: M \rightarrow \mathbb{R}$ is differentiable, for every $x \in M$ we identify in the usual way the differential $d u(x)$ with the gradient $\nabla u(x)$ by means of the scalar product $g_{x}(\cdot, \cdot)$ on the tangent space $T_{x} M$. In this case we obtain the following extension of Theorem 1.4:

Theorem 5.4. Let $(M, g)$ be a Riemannian manifold of dimension $d \geq 2$, consider an open subset $\Omega$ of $M$, and let $F: T \Omega \rightarrow \mathbb{R}$ be a continuous function. Suppose that the following conditions hold:

(A) There exists a $\mathcal{C}^{1}$ function $u_{0}: M \rightarrow \mathbb{R}$, such that $F\left(x, \nabla u_{0}(x)\right) \leq 0$, for every $x \in \Omega$.

(B) There exists a locally bounded function $\rho: \Omega \rightarrow(0, \infty)$ such that, for every $x \in \Omega$, the set $B(x)=\left\{v \in T_{x} M: F(x, v) \leq 0\right\}$ is contained in the ball of center 0 and radius $\rho(x)$ in $T_{x} M$.

Then there exists a differentiable function $u: M \rightarrow \mathbb{R}$ such that:

1. $u \geq u_{0}$ on $M, u=u_{0}$ on $\Omega^{c}$ and $\nabla u=\nabla u_{0}$ on $\Omega^{c}$.

2. $F(x, \nabla u(x)) \leq 0$ for every $x \in \Omega$.

3. $F(x, \nabla u(x))=0$ for almost every $x \in \Omega$.

Proof. We are going to see that the conditions of Theorem 5.1 are satisfied. Fix $x_{0} \in \Omega$ and consider a chart $(W, \varphi)$ in $M$ with $x_{0} \in W$. Associated to this chart, consider the natural diffeomorphism

$$
\Phi: T W \rightarrow \varphi(W) \times \mathbb{R}^{d}
$$

given by $\Phi(x, v)=(\varphi(x), d \varphi(x)(v))$. Choose a compact neighborhood $V^{x_{0}}$ of $x_{0}$ contained in $W$ and $R>0$ such that for every $x \in V^{x_{0}}$ the set 
$B(x)=\left\{v \in T_{x} M: F(x, v) \leq 0\right\}$ is contained in the closed ball of center 0 and radius $R$ in $T_{x} M$. Now set

$$
r=\inf \left\{\|v\|_{x}: x \in V^{x_{0}} ;\|d \varphi(x)(v)\|_{\mathbb{R}^{d}}=1\right\} .
$$

By compactness, it is clear that $r>0$. For every $x \in V^{x_{0}}$ and every $v \in T_{x} M$, we have

$$
\|d \varphi(x)(v)\|_{\mathbb{R}^{d}} \leq \frac{1}{r}\|v\|_{x}
$$

Therefore

$$
\begin{gathered}
B\left(V^{x_{0}}\right)=\left\{(x, v) \in T M: x \in V^{x_{0}} ; F(x, v) \leq 0\right\} \\
\subset\left\{(x, v) \in T M: x \in V^{x_{0}} ;\|v\|_{x} \leq R\right\} \subset \Phi^{-1}\left(\varphi\left(V^{x_{0}}\right) \times \bar{B}\left(0 ; \frac{R}{r}\right)\right) .
\end{gathered}
$$

It follows that $B\left(V^{x_{0}}\right)$ is a compact subset of $T M$.

Corollary 5.5. Let $(M, g)$ be a Riemannian manifold of dimension $\geq 2$ and let $\Omega$ be an open subset of $M$. Then there exists a differentiable function $u: M \rightarrow \mathbb{R}$ such that $\left.u\right|_{\Omega^{c}} \equiv 0$ and $\|\nabla u(x)\|_{x}=1$ for almost every $x \in \Omega$.

Acknowledgements. This research was carried out during a visit of the second author at the University of Bordeaux 1, to which thanks are given. Especially, it is a pleasure to thank the Laboratoire de Mathématiques of this University for its hospitality.

\section{References}

[1] Azagra, D., Ferrera, J. And López-Mesas, F.: Nonsmooth analysis and Hamilton-Jacobi equations on Riemannian manifolds. J. Funct. Anal. 220 (2005), no. 2, 304-361.

[2] Barles, G.: Solutions de viscosité des équations de Hamilton-Jacobi. Mathématiques el Applications 17. Springer-Verlag, Paris, 1994.

[3] Benameur, M. T.: Triangulations and the stability theorem for foliations. Pacific J. Math. 179 (1997), no. 2, 221-239.

[4] Buczolich, Z.: Solution to the gradient problem of C.E. Weil. Rev. Mat. Iberoamericana 21 (2005), no. 3, 889-910.

[5] Crandall, M. G., Ishit, H. and Lions, P. L.: User's guide to viscosity solutions of second order partial differential equations. Bull. Amer. Math. Soc. (N.S.) 27 (1992), no. 1, 1-67.

[6] Deville, R. And Matheron, É.: Infinite games, Banach space geometry and the eikonal equation. Proc. Lond. Math. Soc. (3) 95 (2007), no. 1, 49-68. 
[7] Fathi, A. And Siconolfi, A.: Existence of $C^{1}$ critical subsolutions of the Hamilton-Jacobi equation. Invent. Math. 155 (2004), no. 2, 363-388.

[8] MalÝ, J. And ZelenÝ, M.: A note on Buczolich's solution of the Weil gradient problem: a construction based on an infinite game. Acta Math. Hungar. 113 (2006), no. 1-2, 145-158.

[9] Mantegazza, C. And Mennucci, A. C.: Hamilton-Jacobi Equations and Distance Functions on Riemannian Manifolds. Appl. Math. Optim. 47 (2003), no. 1, 1-25.

[10] Weil, C. E.: On properties of derivatives. Trans. Amer. Math. Soc. 114 (1965), 363-376.

[11] Whitney, H.: Geometric integration theory. Princeton University Press. Princeton, N.J., 1957.

Recibido: 12 de julio de 2007

Robert Deville

Laboratoire de Mathématiques

Université de Bordeaux I

France

Robert.Deville@math.u-bordeaux1.fr

Jesús A. Jaramillo

Departamento de Análisis Matemático

Universidad Complutense de Madrid

28040 Madrid, Spain

jaramil@mat.ucm.es

The second author was supported in part by DGES (Spain) MTM2006-03531. 